\title{
Transverse rectification of disorder-induced fluctuations in a driven system
}

\author{
Alejandro B. Kolton ${ }^{1}$ \\ ${ }^{1}$ Université de Genève, DPMC, 24 Quai Ernest Ansermet, CH-1211 Genève 4, Switzerland *
}

\begin{abstract}
We study numerically the overdamped motion of particles driven in a two dimensional ratchet potential. In the proposed design, of the so-called geometrical-ratchet type, the mean velocity of a single particle in response to a constant force has a transverse component that can be induced by the presence of thermal or other unbiased fluctuations. We find that additional quenched disorder can strongly enhance the transverse drift at low temperatures, in spite of reducing the transverse mobility. We show that, under general conditions, the rectified transverse velocity of a driven particle fluid is equivalent to the response of a one dimensional flashing ratchet working at a drive-dependent effective temperature, defined through generalized Einstein relations.
\end{abstract}

PACS numbers: 74.25.Qt,05.40.-a,05.45.-a,05.60.Cd

The idea of generating a directed dissipative transport in a system kept out of thermal equilibrium only by unbiased perturbations has motivated an outburst of experimental and theoretical works in the last years. The ratchet effect is indeed of interest, both for applications and modelling, in very diverse systems, ranging from biological motors,$\frac{1}{=}$ colloidal matter,$\frac{2}{2}$ granular matter, $\stackrel{3}{=}$ vortex matter in superconductors,,$\frac{4}{\underline{4}}$ Josephson junction arrays, $\frac{\sqrt[5]{5}}{5}$ atoms in optical traps,$\frac{6}{\underline{6}}$ electrons in semicon-

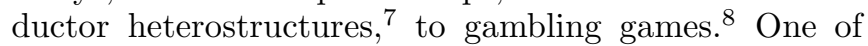
the simplest models is the "flashing ratchet", where a directed motion of a Brownian particle (i.e. breaking of the detailed balance condition) is obtained by coupling it to a pulsating asymmetric-periodic potential. The identification of the essential physical ingredients for the effect shows that a large variety of ratchets and rectification mechanisms can be realized 9

Recently, there has been a growing interest in the socalled geometrical ratchets since they can be used as continuous "molecular sieves" to separate particles experimentally (such as macromolecules or mesoscopic objects), according to its physical properties. These devices are typically two-dimensional systems containing a periodic array of asymmetric obstacles. By driving the particles through the array an average lateral drift appears, as transverse diffusive motion is rectified by the collisions with the asymmetric obstacles. Different types of geometrical ratchets have been analyzed in the literature, both experimentally $\frac{10}{10}$ and theoretically 11 The effect of additional quenched disorder in these two-dimensional systems has not been discussed yet, though interesting anomalous transport properties of one-dimensional disordered ratchet systems were reported ${ }^{12}$ Such a study is not only relevant for applications where disorder can not be avoided, but it is also an interesting and challenging issue. The driven motion of particles in a disordered substrate yields a non-trivial hydrodynamics. The currentdriven motion of vortices in type II superconductors is a prominent example, where disorder, apart from reducing dissipation, is responsible for marked non-equilibrium transport and magnetic properties ${ }^{13}$ On the other hand, already the simpler case of driven non-interacting Brownian particles in two dimensions displays complex phe- (a)

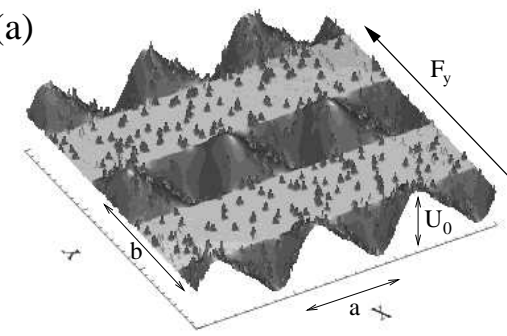

(b)

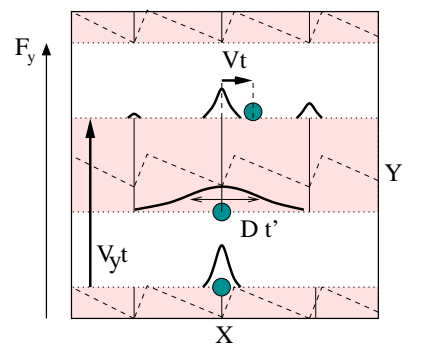

FIG. 1: (a) Ratchet potential with disorder. (b) Schematics of the transverse rectification mechanism (top view). Particles move with an average velocity $V_{y}$ in the direction of the applied force $F_{y}$. In the white regions the interaction with the (attractive or repulsive) centers and with the thermal bath induces diffusion in the non-driven direction In the shaded regions a periodic-asymmetric potential tends to localize particles at its minima. An average transverse shift (see circles) is produced at a rate $V$.

nomena. While diffusion is anomalous at equilibrium, 14 under a finite drive diffusion becomes normal in the comoving frame, with anisotropic and velocity-dependent diffusion constants and mobilities $\frac{15}{15}$ Moreover, a disordered substrate can provide alone a local or global ratchet effect, such as the generation of large-scale vorticity in the probability current by driving particles with an uniform alternate drive,, 16 and the net directed motion produced by driving the particles with crossed ac-drives ${ }^{17}$

In this paper we investigate the effect of quenched disorder in a simple geometrical ratchet design, under an uniform and constant driving force. We find that disorder can strongly enhance the transverse drift both for noninteracting and interacting particles, thus improving the 
performance of the device for applications. We show that the transverse velocity of a driven fluid is equivalent to the response of a one-dimensional flashing ratchet working at a drive-dependent effective temperature, defined through generalized fluctuation-dissipation relations.

Let us consider the overdamped motion of particles in a two dimensional potential like the one depicted in Fig 1 The equation of motion of a particle in position $\mathbf{R}_{i}$ is:

$$
\eta \frac{d \mathbf{R}_{i}}{d t}=-\nabla_{i}\left[\sum_{j \neq i} V\left(R_{i j}\right)+U\left(\mathbf{R}_{i}\right)\right]+\mathbf{F}+\zeta_{i}(t),
$$

where $R_{i j}=\left|\mathbf{R}_{i}-\mathbf{R}_{j}\right|$ is the distance between particles $i, j, R_{i p}$ is the distance between the particle $i$ and a site at $\mathbf{R}_{p}, \eta$ is the friction, and $\mathbf{F}=F_{y} \hat{y}$ is the driving force. The effect of a thermal bath at temperature $T$ is given by the stochastic force $\boldsymbol{\zeta}_{i}(t)$, satisfying $\left\langle\zeta_{i}^{\mu}(t)\right\rangle=0$ and $\left\langle\zeta_{i}^{\mu}(t) \zeta_{j}^{\mu^{\prime}}\left(t^{\prime}\right)\right\rangle=2 \eta k_{B} T \delta(t-$ $\left.t^{\prime}\right) \delta_{i j} \delta_{\mu \mu^{\prime}}$, where $\langle\ldots\rangle$ denotes average over the ensemble of $\boldsymbol{\zeta}_{i}$. For concreteness we consider a logarithmic repulsive particle-particle interaction $V(r)=-A_{v} \ln (r)$ which corresponds for instance to the vortex-vortex interaction in 2D thin film superconductors ${ }^{19}$. Particles interact with the quenched potential $U(\mathbf{R})=$ $U_{R}(\mathbf{R})+U_{p}(\mathbf{R}) . U_{R}$ is a ratchet potential with the form, $U_{R}(\mathbf{R})=\frac{a}{2 \pi} F_{R}(Y) G_{R}(X)$, where $X \equiv \mathbf{R} . \hat{x}, Y \equiv \mathbf{R} . \hat{y}$, $G_{R}(X)=\sin (2 \pi X / a)+0.25 \sin (4 \pi X / a)$ and $F_{R}(y)=$ $U_{0} \cos (2 \pi y / b) \Theta[\cos (2 \pi y / b)]$, with $\Theta$ the Heaviside function. This ratchet potential is similar to a periodic array of obstacles, asymmetrical around the $x$-axis but symmetrical around the $y$-axis, as the ones considered in Ref. 11 Disorder is short-range correlated, and it is modeled as a random distribution of centers such that $U_{p}\left(\mathbf{R}_{i}\right)=\sum_{p} A_{p} e^{-\left(R_{i p} / r_{p}\right)^{2}}$, where $R_{i p}=\left|\mathbf{R}_{i}-\mathbf{R}_{p}\right|$ is the distance between the particle $i$ and a center at $\mathbf{R}_{p}$. Centers can be either attractive $A_{p}<0$ (wells) or repulsive $A_{p}>0$ (humps) or a combination of both. We solve Eq1 numerically by using the method of Ref ${ }^{18}$. Length is normalized by $r_{p}$, energy by $2 A_{p}$, and time by $\tau=\eta r_{p}^{2} /\left|2 A_{p}\right|$. We consider $N=60$ particles and $N_{p}$ pinning centers in a rectangular box of size $L_{x} \times L_{y}$ and periodic boundary conditions, with $L_{y}=100, L_{x}=20 \sqrt{3} L_{y}$. We average calculated properties over 500 disorder realizations.

We start by discussing the simplest case of noninteracting particles, $A_{v}=0$, without disorder, $N_{p}=0$, and with a ratchet potential of amplitude $U_{0}=1$. The dashed-lines of Fig 2(a) show the transverse drift rate $V \equiv\left\langle\frac{1}{N} \sum_{i} \frac{d X_{i}}{d t}\right\rangle$ at $T=0.05$ as a function of the longitudinal velocity $V_{y} \equiv\left\langle\frac{1}{N} \sum_{i} \frac{d Y_{i}}{d t}\right\rangle$. We see that the transverse velocity $V$ increases from zero, has a maximun $V \sim 0.0075$ at $V_{y} \sim 0.5$, and decays to zero at large longitudinal velocity. Since $V=0$ at $T=0$, the average directed transverse motion observed is induced by the thermal noise. This rectification effect is easy to understand, and Fig (b) illustrates the mechanism, where the transverse diffusion constant is $D=2 T$ if there is no disorder nor inter-particle interactions. For our discussion it is useful to make explicit the connection between the type of response shown in Fig 2(a) and the one of a flashing ratchet. If $F_{y}$ is large, the mean velocity in the driven direction $V_{y} \equiv\left\langle\frac{d Y}{d t}\right\rangle$ is $V_{y} \sim F_{y}-O\left(F_{y}^{-1}\right)$ and longitudinal fluctuations are much smaller (by a factor $\left.O\left(F_{y}^{-1}\right)\right)$ than transverse fluctuations $\frac{15}{x}$ At $T=0$, the equation of motion for the coordinate $X$ of a particle located at $\mathbf{R}=X \hat{x}+Y \hat{x}$ can be thus written as

$$
\frac{d X}{d t} \approx-F_{R}\left(V_{y} t\right) G_{R}^{\prime}(X)
$$

Since $F_{R}\left(V_{y} t\right)=U_{0} \cos \left(2 \pi V_{y} t / b\right) \Theta\left[\cos \left(2 \pi V_{y} t / b\right)\right], \quad X$ feels the ratchet potential $G_{R}(X)$ switching on and off periodically with time periods $\tau_{\text {on }}=\tau_{\text {off }}=b / 2 V_{y}$. At small drives the mechanism is the same although $\tau_{\text {on }}$ becomes increasingly larger than $\tau_{\text {off }}$ since the wells of $U_{R}(X, Y)$ delay the motion in the $y$ direction. (see Fig 1). The mapping to a flashing ratchet explains the observed directed transverse motion with $V>0$ when $T>0$ and can be thus used as an effective model to explain all the features of the response shown in Fig 2(a).

The effect discussed so far is similar to the one described in Ref. 11 Let us now add disorder, by putting $N_{p}=2000$ randomly located pinning sites. The resulting response is shown in Fig 2 (a) (symbols). As we can see, disorder strongly enhances the rectification at intermediate and large longitudinal velocity and also broaden the range of $V_{y}$ where response is appreciable, with respect to the clean case. In addition, we find that at intermediate and large $V_{y}, V$ is finite even in the $T=0$ limit, since disorder induces transverse diffusion when $V_{y}>0$, even in the absence of thermal fluctuations. Finally, let us now turn-on the repulsive interaction between particles. In Fig 2(b) we show $V$ as a function of $V_{y}$, for different values of the repulsion strength $A_{v}$. In the inset of Fig 2(b) we see that the maximun response $V_{\max }$ is almost constant with $A_{v}$ up to values $A_{v} \sim 0.2$ where a slow decay starts, but it is larger than the response of the clean system up to $A_{v}=2$. The decay of the response at large $A_{v}$ is explained by the decrease of transverse wandering due to increasingly correlated collective motion ${ }^{19}$ In Fig 2(b) we show that the response for purely attractive pinning centers $A_{p}=-1$ is smaller than for repulsive centers $A_{p}>1$ for small values of $V_{y}$, but indistinguishable for larger values of $V_{y}$. This is due to the fact that, at the density of centers considered, attractive centers are more effective to pin particles than humps, since the latter can provide two-dimensional pinning only by forming rare geometric traps. However at a density $N_{p} / L_{x} L_{y} \sim 1 / r_{p}^{2}$ all these differences disappear completely.

In order to understand the rectification characteristics described above it is instructive to study, separately, the motion of particles in the purely disordered case, without the ratchet potential (i.e. $U_{0}=0$ ). For simplicity we consider only the case of non-interacting particles, but we expect similar results for interacting particles in the dynamical regimes where transverse diffusion is non-zero $\stackrel{18,20}{ }$ We analyze in detail the non-equilibrium transverse fluctuations as a function of $F_{y}$, since they 


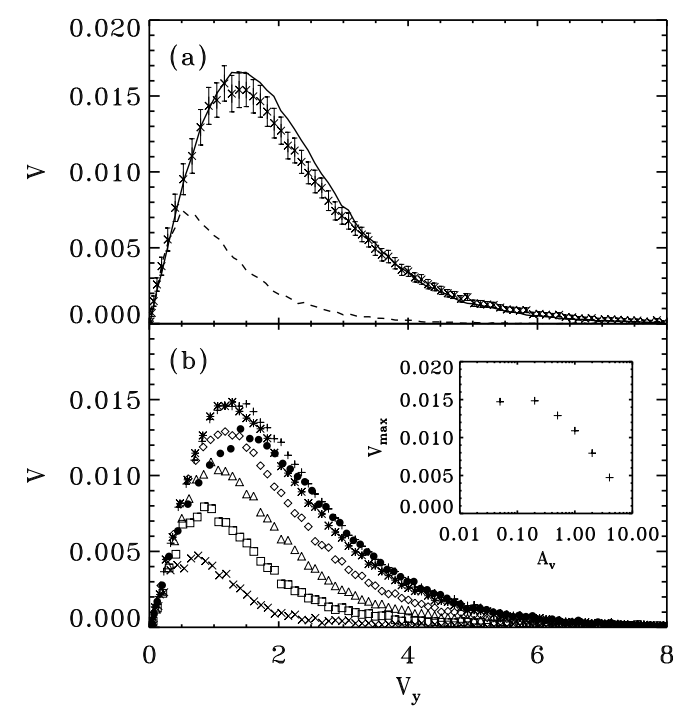

FIG. 2: Transverse velocity $V$ vs longitudinal velocity $V_{y}$. (a) Symbols correspond to the disordered system at $T=0.05$, the dashed-line to the clean system at $T=0.05$ and the solid-line to the clean system at an effective temperature $T_{\text {eff }}\left(V_{y}, T\right)$. (b) $V$ vs $V_{y}$ for different interaction strengths $A_{v}: A_{v}=0.05$ $(+), A_{v}=0.2(*), A_{v}=0.5(\diamond), A_{v}=1(\triangle), A_{v}=2(\square)$, $A_{v}=5(\times)$. $(\bullet)$ symbols correspond to purely attractive pinning centers, with $A_{p}=-0.5$, at $T=0.05$. Inset: maximun rectification as a function of $A_{v}$.

affect directly the rectification in the presence of the ratchet potential. Following Ref ${ }^{18}$ we define the observables $O(t)=\frac{1}{N_{v}} \sum_{i=1}^{N_{v}} s_{i} X_{i}(t)$ and $\tilde{O}(t)=\sum_{i=1}^{N_{v}} s_{i} X_{i}(t)$, where $s_{i}=-1,1$ are random numbers with $\overline{s_{i}}=0$ and $\overline{s_{i} s_{j}}=\delta_{i j}$. The quadratic mean displacement can be written as $\Delta\left(t, t_{0}\right) \equiv \frac{1}{N_{v}} \sum_{i=1}^{N_{v}}\left\langle\left|X_{i}(t)-X_{i}\left(t_{0}\right)\right|^{2}\right\rangle=$ $C(t, t)+C\left(t_{0}, t_{0}\right)-2 C\left(t, t_{0}\right)$, with $C\left(t, t_{0}\right)=\overline{\left\langle O(t) \tilde{O}\left(t_{0}\right)\right\rangle}$. The integrated response function $\chi$ for the observable $O$ is obtained by applying a perturbative force $\mathbf{f}_{i}=\epsilon s_{i} \hat{x}$ at time $t_{0}$ and keeping it constant for all subsequent times on each particle, $\chi\left(t, t_{0}\right)=\lim _{\epsilon \rightarrow 0} \frac{1}{\epsilon}\left[{\overline{\langle O(t)\rangle_{\epsilon}}}_{\epsilon}-{\overline{\langle O(t)\rangle_{\epsilon=0}}}_{\text {. }}\right.$. In the steady state $\Delta\left(t, t_{0}\right)=\Delta\left(t-t_{0}\right)$ and $\chi\left(t, t_{0}\right)=$ $\chi\left(t-t_{0}\right)$ and in particular at equilibrium the fluctuationdissipation theorem (FDT) imposes $\chi(t)=\Delta(t) / 2 T$. When $F_{y}>0$ the system is out of equilibrium and the FDT does not hold. We will show however, that generalized fluctuation-dissipation relations can still be defined for our system. In the long time limit we find $\Delta(t) \sim D t$ and $\chi(t) \sim \mu t$ thus allowing us to define the transverse diffusion constant $D$ and the transverse mobility $\mu$. These two quantities depend on the longitudinal driving force as shown in Fig 3 (b). $D$ is non-monotonic, has a peak at $F_{y} \sim 1.5$, and decays approximately as a power law towards the equilibrium value without disorder $2 T$ for large forces. This behavior can be understood by considering the effective transverse random walk induced only by the collisions with the pinning centers at

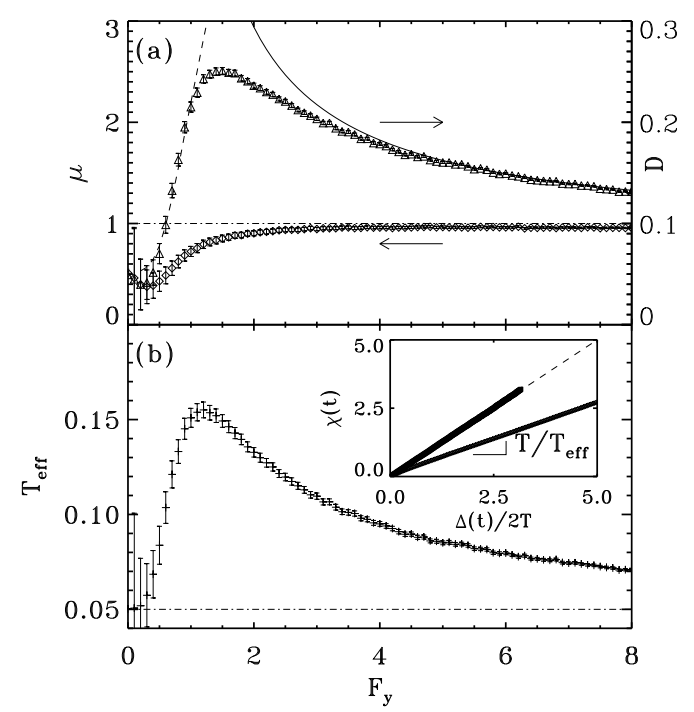

FIG. 3: Motion in a purely disordered potential as function of $F_{y}$. (a) Transverse ( $x$ direction) diffusion constant $D$ and mobility $\mu$. Dashed and solid lines indicate asymptotic forms of $D$. (b) Effective temperature $T_{\text {eff }} \sim D / 2 \mu$. The dasheddotted line indicates the thermal bath temperature, $T=0.05$. The inset shows that $T_{\text {eff }}$ satisfies a generalized fluctuationdissipation relation between the integrated response $\chi(t)$ and the quadratic mean displacement $\Delta(t)$. The dashed line corresponds to the equilibrium fluctuation-dissipation relation, which is valid only at short times $t \lesssim \xi / V_{y}$. Upper symbols correspond to $F_{y}=0.0$, and lower symbols to $F_{y}=4.0$.

$T=0$, and by simple heuristic arguments is possible to find the asymptotic forms $D \sim n_{p} r_{p}^{3} V_{y}$ at small $V_{y}$ and $D \sim n_{p} r_{p} A_{p}^{2} / V_{y}$ at large $V_{y}, \frac{15}{=}$ indicated in Fig 3 (b). At large $F_{y}$ the transverse mobility $\mu$ approaches the equilibrium value without disorder, $\mu=1$ (independent of $T$ ). At small $F_{y}, \mu$ decreases due to trapping and its value at the limit $F_{y} \rightarrow 0$ is controlled by $T$. In the inset of Fig 3 (c) we show the parametric plot of $\chi(t)$ vs $\Delta(t) / 2 T$ for $F_{y}=0$ (equilibrium) and $F_{y}=4.0$ (out of equilibrium). We see that the equilibrium FDT holds for $F_{y}=0$ as expected. For $F_{y}=4.0$ (and in general for $F_{y}>0$ ) we observe instead that the FDT holds only at very short time scales, $t \lesssim r_{p} / V_{y}$, but it is violated at long times. The type of violation observed can be quantified using the notion of time-scale dependent "effective temperatures" introduced by Cugliandolo, Kurchan and Peliti ${ }^{21}$ Following Ref ${ }^{18}$ we define a velocity-dependent transverse effective temperature $T_{\text {eff }}$ from the slope shown in the inset of Fig 3(c). At long times this implies the generalized Einstein relation $T_{\text {eff }} \sim D / 2 \mu$ in the non-driven direction. In Fig 3(c) we see that $T_{\text {eff }}$ follows closely $D$ except at low forces where $\mu$ decreases towards the value $\mu \sim 0.5$ at very low forces. For interacting particles similar results for $T_{\text {eff }}$ were obtained at low and intermediate forces, in the plastic and smectic regimes of motion $\stackrel{19}{ } \mathrm{At}$ large forces and small temperatures however, the forma- 
tion of pinned rough channels for particle motion ${ }^{13}$ leads to a transverse freezing of the moving vortex fluid ${ }^{19}$ at $T=0$. At this dynamical transition $D$ and $\mu$ are strongly reduced, and vanish at $T=0$ strictly 15.18

The fluctuating motion in a purely disordered substrate and the connection between the response of a geometrical and a flashing ratchet discussed above motivate a simple model that can be used to describe the rectification response of the disordered geometrical ratchet. We propose the following equation for the transverse motion of non-interacting particles,

$$
\frac{1}{\mu\left(V_{y}\right)} \frac{d X_{i}}{d t} \approx-F_{R}\left(V_{y} t\right) G_{R}^{\prime}\left(X_{i}\right)+\zeta_{\mathrm{eff}}^{i}(t) .
$$

where we have replaced $Y_{i} \sim V_{y} t$ as before, the pinning force by an effective thermal noise $\zeta_{\text {eff }}^{i}(t)$ and the bare mobility by $\mu\left(V_{y}\right)$. We use that $\left\langle\zeta_{\text {eff }}^{i}(t)\right\rangle=0$ and $\left\langle\zeta_{\text {eff }}^{i}(t) \zeta_{\text {eff }}^{j}\left(t^{\prime}\right)\right\rangle=\left(2 T_{\text {eff }}\left(V_{y}\right) / \mu\left(V_{y}\right)\right) \delta\left(t-t^{\prime}\right) \delta_{i j}$ where $T_{\text {eff }}\left(T, V_{y}\right)$ and $\mu\left(T, V_{y}\right)$ are the ones shown in Fig 3 (a). Eq. 3 therefore models transverse motion in a coarsegrained way (in time and space), satisfying the generalized fluctuation-dissipation relation shown in the inset of Fig 3 b) (in the absence of the ratchet potential). By construction, the assumptions of the model are that (i) transverse forces are small compared with the longitudinal drive $F_{y}$ and (ii), the particle motion is incoherent at the length scales of the ratchet potential. In Fig 2(a) we see that the transverse drift generated by this model is close to the one of the full model, for the parameters analyzed in this paper. The rectification characteristics of the two-dimensional geometrical ratchet are therefore well described by the one-dimensional flashing-ratchet described by Eq[3 working at the effective temperature $T_{\text {eff }}\left(T, V_{y}\right)$ and friction $\mu^{-1}\left(T, V_{y}\right)$, determined by the disorder and the longitudinal velocity. Using this model the enhancement of the rectification observed in Fig[2(a) can be simply attributed to the fact that $T_{\text {eff }}>T$, but with $T_{\text {eff }}$ still smaller than the optimal temperature for rectification of the effective pulsating ratchet Eq 3 is also expected to work for interacting particles by using the respective $T_{\text {eff }}\left(T, V_{y}\right)$ and $\mu\left(T, V_{y}\right)$ except at low $T$ and large $F_{y}$ where condition (ii) can be violated since particles become correlated over long times and distances. ${ }^{20}$

In conclusion, we have studied numerically the effect of quenched disorder in a geometrical ratchet. We find that disorder enhances the transverse rectified velocity of a driven fluid. If particle motion is incoherent at the scale of the ratchet potential the response can be simply described by a one-dimensional flashing ratchet working at a disorder-induced, drive-dependent effective mobility and temperature, satisfying generalized Einstein relations. This effect can be used experimentally to enhance and control the performance of geometrical ratchets.

We acknowledge discussions with L.F. Cugliandolo, D. Domínguez, T. Giamarchi, V.I. Marconi, and A. Rosso. This work was supported in part by the Swiss National Fund under Division II.
* Present address: Dept. de Física Atómica, Molecular y Nuclear, Universidad Complutense de Madrid, 28040 Madrid, Spain.

1 R. D. Astumian and M. Bier, Phys. Rev. Lett. 72, 1766 (1994); R. D. Astumian, Science 276, 917 (1997); Jülicher, A. Adjari, and J. Prost, Rev. Mod. Phys. 69, 1269 (1997).

2 C. Marquet et al., Phys. Rev. Lett. 88, 168301 (2002); S. H. Lee et al., Phys. Rev. Lett. 94, 110601 (2005); D. Babic and C. Bechinger, Phys. Rev. Lett. 94, 148303 (2005); A. Libál et al., Phys. Rev. Lett. 96, 188301 (2006).

3 Z. Farkas et al., Phys. Rev. E 60, 7022 (1999); J. F. Wambaugh, C. Reichhardt, and C. J. Olson, Phys. Rev. E 65, 031308 (2002); D. van der Meer et al., Phys. Rev. Lett. 92, 184301 (2004).

${ }^{4}$ C. S. Lee et al., Nature (London) 400, 337 (1999); J. F. Wambaugh et al., Phys. Rev. Lett. 83, 5106 (1999); M. B. Hastings, C. J. Olson Reichhardt, and C. Reichhardt, Phys. Rev. Lett. 90, 247004 (2003); C. J. Olson et al., Phys. Rev. Lett. 87, 177002 (2001); B. Y. Zhu et al., Phys. Rev. B 68, 014514 (2003); R. Wördenweber, P. Dymashevski, and V. R. Misko, Phys. Rev. B 69, 184504 (2004); J. E. Villegas et al., 71, 024519 (2005).

${ }^{5}$ F. Falo et al., Europhys. Lett. 45, 024519 (1999); E. Trias et al., Phys. Rev. E 61, 2257 (2000); G. Carapella and G. Costabile, Phys. Rev. Lett. 87, 077002 (2001); D. E. Shalóm and H. Pastoriza, Phys. Rev. Lett. 94, 177001 (2005); V. I. Marconi, Physica C 437, 195 (2006);
A. Sterck, R. Kleiner, and D. Koelle, Phys. Rev. Lett. 95, 177006 (2005).

${ }^{6}$ E. Lundh and M. Wallin, Phys. Rev. Lett. 94, 110603 (2005); R. Gommers, S. Bergamini, and F. Renzoni, Phys. Rev. Lett. 95, 073003 (2005); M. Schiavoni et al., Phys. Rev. Lett. 90, 094101 (2003); R. Gommers, S. Denisov, and F. Renzoni, Phys. Rev. Lett. 96, 240604 (2006).

7 H. Linke et al., Science 286, 2314 (2003).

8 J. M. R. Parrondo and L. Dinís, Contemporary Physics 45, 147 (2004).

9 P. Reimann, Phys. Rep. 361, 57 (2002); R. D. Astumian and P. Hänggi, Physics Today 55, 33 (2002).

10 A. van Oudenaarden and S. G. Boxer, Science 285, 1046 (1999).

11 D. Ertas, Phys. Rev. Lett. 80, 1548 (1998); T. A. J. Duke and R. H. Austin, Phys. Rev. Lett. 80, 1552 (1998); I. Derényi and R.D. Astumian, Phys. Rev. E 58, 7781 (1998); M. Bier et al., M. Kostur, 61, 7184 (2000); M. Kostur and L. Shimansky-Geier, Phys. Lett. A 265, 337 (2000); C. Keller, F. Marquardt, and C. Bruder, Phys. Rev. E 65, 041927 (2002); S. Savel'ev et al., Phys. Rev. B 71, 214303 (2005).

12 T. Harms and R. Lipowsky, Phys. Rev. Lett. 79, 2895 (1997); F. Marchesoni, Phys. Rev. E 56, 2492 (1997); M. N. Popescu et al., Phys. Rev. Lett. 85, 3321 (2000).

13 T. Giamarchi and S. Bhattacharya, in High Magnetic Fields: Applications in Condensed Matter Physics and 
Spectroscopy, edited by C. Berthier et al. (Springer-Verlag, Berlin, 2002), p. 314, cond-mat/0111052.

14 J.-P. Bouchaud and A. Georges, Phys. Rep 195, 127 (1990).

15 A. B. Kolton, Physica C 437, 026112 (2006).

16 M. A. Makeev, I. Derenyi, and A.-L. Barabasi, Phys. Rev. E 71, 026112 (2005).

17 C. Reichhardt and C. J. Olson Reichhardt, Phys. Rev. E
73, 011102 (2006).

18 A. B. Kolton et al., Phys. Rev. Lett. 89, 227001 (2002).

19 A. B. Kolton, D. Domínguez, and N. Grønbech-Jensen, Phys. Rev. Lett. 83, 3061 (1999).

20 A. B. Kolton, to be published.

21 L. F. Cugliandolo, J. Kurchan, and L. Peliti, Phys. Rev. E 55, 3898 (1997). 